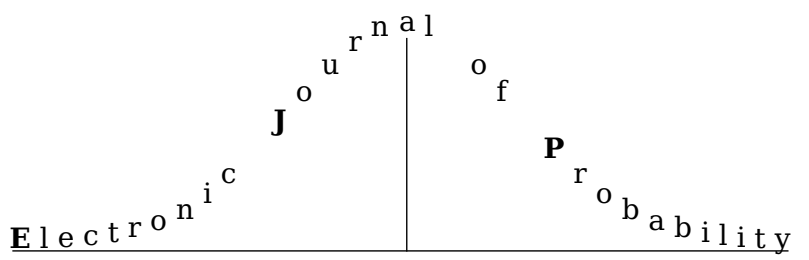

Electron. J. Probab. 26 (2021), article no. 63, 1-16.

ISSN: 1083-6489 https://doi.org/10.1214/21-EJP609

\title{
A note on recurrence of the Vertex reinforced jump process and fractional moments localization*
}

\author{
Andrea Collevecchio ${ }^{\dagger} \quad{\text { Xiaolin } \text { Zeng }^{\ddagger}}^{\ddagger}$
}

\begin{abstract}
We give a simple proof for recurrence of vertex reinforced jump process on $\mathbb{Z}^{d}$, under strong reinforcement. Moreover, we show how the previous result implies that linearly edge-reinforced random walk on $\mathbb{Z}^{d}$ is recurrent for strong reinforcement. Finally, we prove that the $H^{(2 \mid 2)}$ model on $\mathbb{Z}^{d}$ localizes at strong disorder. Even though these results are well-known, we propose a unified approach, which also has the advantage to provide shorter proofs, and relies on estimating fractional moments, introduced by Aizenman and Molchanov.
\end{abstract}

Keywords: vertex-reinforced jump process; localization; recurrence.

MSC2020 subject classifications: $60 \mathrm{~K} 35$.

Submitted to EJP on April 18, 2020, final version accepted on March 24, 2021.

\section{Introduction}

The Vertex Reinforced Jump Process (VRJP) is a continuous time self-interacting process. It was first studied by Davis and Volkov ([DV02] and [DV04]) on $\mathbb{Z}$. See also [Col09, BS10] and [CZ18] for a study of VRJP on trees, and [RN18] for super-linear VRJP. Recent studies [ST15a] revealed a close relation between VRJP and linearly edge reinforced random walks (ERRW, introduced by Coppersmith and Diaconis [CD87]). Moreover, VRJP is also related to a supersymmetric hyperbolic sigma model (introduced by Zirnbauer [Zir91]), called the $H^{(2 \mid 2)}$-model, studied in [DS10, DSZ10]. The latter is a toy model for the study of Anderson transition. The paper [STZ17] introduced a random operator which is naturally related to these objects.

In [ST15a] and [ACK14], were given two different proofs of the fact that the ERRW are recurrent under strong reinforcement on $\mathbb{Z}^{d}$. These were long-standing open problems in the field. We will give yet an alternative short proof, using a unifying approach built

${ }^{*}$ A.C. was supported by ARC grant DP180100613 and Australian Research Council Centre of Excellence for Mathematical and Statistical Frontiers (ACEMS) CE140100049. X.Z. was supported by ERC starting grant 678520 and supported in part by Israel Science Foundation grant 861/15.

${ }^{\dagger}$ School of Mathematics Monash University, Australia. E-mail: andrea. collevecchio@monash.edu

${ }^{\ddagger}$ IRMA, Université de Strasbourg, France. E-mail: zeng@math. unistra. fr 
VRJP and fractional moment localization

on ideas from [AM93]. In particular, we use the fact that ERRW is a time change of VRJP with random i.i.d. conductance to prove recurrence of ERRW on $\mathbb{Z}^{d}$ when the reinforcement is strong enough.

\section{The model}

We define VRJP as follows. Let $\mathcal{G}=(V, E, W, \theta)$ be a non-directed locally finite weighted graph, where to each edge $e \in E$ is assigned an initial weight $W_{e} \geq 0$ and to each vertex $i$ is assigned an initial weight $\theta_{i}>0$. Moreover, we assume that $\mathcal{G}$ has self-loops, i.e. edges connecting a vertex to itself; each pair of vertices in $V$ can be joined by at most one edge, and two vertices $i, j$ are neighbors, denoted by $i \sim j$, if they are joined by an edge. In this paper, we mainly focus on $\mathbb{Z}^{d}$ and its sub-graphs, with general weights, possibly random. Denote by $W=\left(W_{e}\right)_{e \in E}$ and $\theta=\left(\theta_{i}\right)_{i \in V}$. For $i \sim j$ we use the notation $W_{i, j}$ for the weight on the edge connecting $i$ and $j$. If $i$ and $j$ are not neighbors, we set $W_{i, j}=0 . \operatorname{VRJP}(W, \theta)$ on $\mathcal{G}$ is a continuous time process that takes values on $V$. This process is denoted by $\mathbf{Y}=\left(Y_{t}\right)_{t \geq 0}$ and starts at $Y_{0}=i_{0} \in V$, where $i_{0} \in V$ is a designated vertex. Conditionally on the past of $\mathbf{Y}$ up to time $t$, and conditionally to $Y_{t}=i$, this process jumps at time $t$ towards $j$ at rate

$$
W_{i, j} L_{j}(t) \quad \text { where } L_{j}(t)=\theta_{j}+\int_{0}^{t} \mathbb{1}_{Y_{u}=j} d u .
$$

In particular, VRJP can only jump among adjacent vertices. It is shown in [ST15a] that after a suitable time change, the VRJP is a mixture of Markov jump processes. Section 3 contains a descriptions of the mixing measure and its very useful properties.

Next, we define the Linearly Edge-Reinforced Random Walk (ERRW). Fix a collection $a=\left(a_{e}\right)_{e \in E}$ of positive real numbers, they are called the initial weights of ERRW. It is a discrete time process, which takes values on $V$, and at each step jumps between nearest neighbors, updating the weights on the edges as follows. Initially to each edge $e$ is assigned a weight $a_{e}$. Each time the process traverses an edge, the weight of that edge is increased by 1 . The probability to traverse a given edge at a given time is proportional to the weight of that edge at that time. We denote $\operatorname{ERRW}(a)$ for such a process.

We use the notation $\operatorname{VRJP}(W)$ to denote VRJP with edge weights $W$ and vertex weights $\theta_{i} \equiv 1$, that is $\operatorname{VRJP}(W, 1)$. It turns out that, as a consequence of Theorem C, or Corollary 1 of [STZ17], the two models $\operatorname{VRJP}(W, \theta)$ and $\operatorname{VRJP}\left(W^{\theta}, 1\right)$ (where $W_{i, j}^{\theta}=W_{i, j} \theta_{i} \theta_{j}$ ) behave the same in terms of recurrence/transience. Moreover, most of our results on infinite graphs assume some ergodicity of the model w.r.t. $\mathbb{Z}^{d}$-translation, that is, for simplicity, we frequently consider constant $W_{i, j} \equiv W$ and $\theta_{i} \equiv \theta$ on $\mathbb{Z}^{d}$, in such case we also have equivalence among the models $\operatorname{VRJP}(W, \theta), \operatorname{VRJP}\left(W^{\theta}, 1\right)$ and $\operatorname{VRJP}(1, \sqrt{W} \theta)$. In particular, considering $\operatorname{VRJP}(W)$ is almost as general as considering $\operatorname{VRJP}(W, \theta)$.

Theorem 2.1. 1. Consider a collection of independent positive random variables $\widetilde{W}=\left\{\widetilde{W}_{e}: e \in E\right\}$. Consider the process $\mathbf{Y}$ defined as follows. Conditionally on $\widetilde{W}$, $\mathbf{Y}$ is $\operatorname{VRJP}(\widetilde{W})$. There exists $\bar{W}_{d} \in(0, \infty]$ such that if

$$
\sup _{e \in E} \mathbb{E}\left[\widetilde{W}_{e}^{1 / 4}\right]<\bar{W}_{d}
$$

then $\mathbf{Y}$ is recurrent ${ }^{1}$.

2. Consider $\operatorname{ERRW}(a)$ on $\mathbb{Z}^{d}$, with $d \geq 1$. There exists $\bar{a}(d) \in(0, \infty)$ such that $\operatorname{ERRW}(a)$ satisfying $\sup _{e \in \mathbb{E}} a_{e} \leq \bar{a}(d)$ is recurrent.

\footnotetext{
${ }^{1}$ By recurrent we mean that the process visit its starting position infinitely often almost surely.
} 
Remark 2.2. In our proof, we provide bounds for $\bar{W}_{d}$. More precisely,

$$
\bar{W}_{d} \geq \frac{\sqrt{\pi}}{\Gamma(1 / 4) 2^{3 / 4} d} .
$$

Corollary 2.3. Consider $\operatorname{VRJP}(W)$ on $\mathbb{Z}^{d}$, where $W=\left(W_{e}\right)_{e \in E}$ is a collection of deterministic weights and $E$ is the edge set of $\mathbb{Z}^{d}$. Let $\bar{W}_{d}$ be as in Theorem 2.1. If $\sup _{e \in E} W_{e}<\bar{W}_{d}^{4}$, then the $\operatorname{VRJP}(W)$ is recurrent.

Moreover, we were able to apply our proof to show localization of a random Schrödinger operator $H(\theta)$ (c.f. definition in Theorem A) connected both to the $H^{(2 \mid 2)}$ model (introduced in [Zir91]) and $\operatorname{VRJP}(1, \theta)$. It is shown in Theorem 1 of [DS10] that, the Green function of $H(\theta)$ at energy level 0 (ground state) decays exponentially when $\theta$ is small enough. The following theorem is slightly more general, as we establish, when $\theta$ is small enough, the exponential decay for the Green function at all energy levels.

Theorem 2.4. Consider the graph $\mathbb{Z}^{d}$ where to each vertex is assigned weight $\theta>0$, and to each edge weight 1 . In this context, denote the operator introduced in Definition 3.3 by $H_{\theta}$ with $\eta=0$. There exists $\theta_{0}$, which depends on $d$ only, such that if $\theta<\theta_{0}$ we have that the operator $H_{\theta}$ is localized, i.e. has a.s. a complete set of orthonormal eigenfunctions, which decay exponentially.

The first part of Theorem 2.1 clearly implies Corollary 2.3. Moreover, Part 2) of Theorem 2.1 is a corollary of part 1). In fact, it relies on a result of Sabot and Tarrès which can be described as follows. If $\mathbf{Y}$ is the process defined in Theorem 2.1 part 1), where $\widetilde{W}=\left(\widetilde{W}_{e}\right)_{e \in E}$ are independent random variables and $\operatorname{Gamma}\left(a_{e}, 1\right)$ distributed; then the skeleton of $\mathbf{Y}$ (that is, the discrete time process associated to $\mathbf{Y}$ ) equals in distribution to $\operatorname{ERRW}(a)$ (proof can be found in Section 4). Hence, we only need to prove Theorem 2.1 part 1 ).

The rest of the paper is organized as follows: In Section 3 we will introduce a family of mixing measures connected with the local times of VRJP and derive some of its properties for later use. In Section 4, as a preparation, we recall the fact that VRJP is a mixture of Markov jump processes, and to be self-contained, we provide proofs. Section 5 is devoted to the proof of recurrence in strong reinforcement, i.e. Theorem 2.1 part 1). Finally, in Section 6, we show that, the operator related to the VRJP is localized in strong disorder, as an application of [AM93].

\section{The multivariate inverse Gaussian distribution}

The aim of this section is to introduce and study the properties of a particular random potential of some Schrödinger operators on finite weighted graphs. This operator is then extended to infinite graphs, in particular $\mathbb{Z}^{d}$.

Consider a finite weighted graph $\mathcal{G}=(V, E, W, \theta)$. For notational reason we identify $V$ with the set $\{1, \ldots, N\}$. Recall that to each unordered pair of vertices $\{i, j\}$ we assign a non-negative weight $W_{i, j}$, which is strictly positive if and only if $i \sim j$, and denote $W=\left(W_{e}\right)_{e \in E}$ for short. Moreover, to each vertex $i \in V$ is assigned a real number $\theta_{i}>0$, and $\theta=\left(\theta_{i}\right)_{i \in V}$.

Definition 3.1. A Schrödinger operator $H_{\beta}$ on the finite weighted graph $\mathcal{G}$ with potential $2 \beta \in \mathbb{R}^{N}$ is an $N \times N$ matrix with the following entries $(1 \leq i, j \leq N)$

$$
H_{\beta}(i, j)=\left\{\begin{array}{ll}
2 \beta_{i} & i=j \\
-W_{i, j} & i \neq j
\end{array} .\right.
$$

For any $\beta \in \mathbb{R}^{N}$, let $[\beta]$ be the diagonal matrix where the $i$-entry of the diagonal is $\beta_{i}$, for $1 \leq i \leq N$. If we denote by $\Delta_{W}$ the weighted incidence matrix, whose entries are 
$\left(W_{i, j}\right)_{i, j \in V}$ (recall that $W_{i, i}=0$ ), we have $H_{\beta}=2[\beta]-\Delta_{W}$. We call $2 \beta$ the potential, and even though this choice might differ with part of the literature, we aim to be consistent with the terminology used in few papers that studied VRJP, e.g. in [STZ17, SZ19]. A main ingredient in the present paper is a random version of $H_{\beta}$, with a particular random potential $\beta$ defined by the following theorem.

The proof of the following theorem is due to Letac and Jacek [LW17] and can also be found in [SZ19]. For the sake of completeness, we included a proof in the Appendix.

Theorem A. Let $H=H_{\beta}$ be defined as in (3.1). If $\theta=\left(\theta_{1}, \ldots, \theta_{N}\right)$ and $\eta=\left(\eta_{1}, \ldots, \eta_{N}\right)$ are vectors with real positive coordinates, then

$$
\int_{H>0} \frac{\prod_{i} \theta_{i}}{\sqrt{(\pi / 2)^{N}}} e^{-\frac{1}{2}\left(\langle\theta, H \theta\rangle+\left\langle\eta, H^{-1} \eta\right\rangle-2\langle\theta, \eta\rangle\right)} \frac{1}{\sqrt{\operatorname{det} H}} \prod_{i \in V} d \beta_{i}=1,
$$

where $\langle\cdot, \cdot\rangle$ is the usual scalar product of $\mathbb{R}^{N},\langle\theta, H \theta\rangle=\sum_{1<i, j<N} H(i, j) \theta_{i} \theta_{j}$, and $\{H>0\}$ is the collection of $\beta$ such that $H_{\beta}$ is positive definite. In particular, the integrand is a probability density and defines the distribution of an $N$-dimensional random vector $\beta$.

The density appearing in the integrand in (3.2) is a multidimensional version of the inverse Gaussian distribution. More precisely, (3.2) is a generalization of the well-known fact, that for any $a, b>0$

$$
\frac{a}{\sqrt{2 \pi}} \int_{0}^{\infty} e^{-\frac{1}{2}\left(a^{2} x+b^{2} / x-2 a b\right)} \frac{1}{\sqrt{x}} d x=1 .
$$

Remark 3.2. The density appearing in Theorem A has a rather long history and also have several names. First of all, it is a generalization of both the Gamma distribution and the Inverse Gaussian (IG) distribution. It is related to the magic formula proposed in [CD87], which is then discussed in [DR06, KR00, MÖR08]. In the meantime, it also appears as a hyperbolic supersymmetric measure in [Zir91, DSZ10, DS10], which is then connected to the magic formula by Sabot and Tarrès in [ST15a]. It is introduced in the above form (with $\eta=0$ ) in [STZ17] and with $\eta \neq 0$ in [SZ19, DMR17a, LW17].

Definition 3.3. We say that $\beta$ is $\nu^{W, \theta, \eta}$ distributed if its density is defined by (3.2). Let $\beta$ be $\nu^{W, \theta, \eta}$ distributed, and $H_{\beta}$ defined as in (3.1). Then $H_{\beta}$ is a random Schrödinger operator (as its potential is random) associated to the weighted graph $\mathcal{G}$. Sometimes, we denote this operator by $H_{W, \theta, \eta}$ to emphasize its dependency on the parameters.

For a subset $V_{1}$ of $V$, let us denote $H_{V_{1}, V_{1}}$ the sub-matrix $(H(i, j))_{i, j \in V_{1}}$ and $\theta_{V_{1}}$ is the sub-vector $\left(\theta_{i}\right)_{i \in V_{1}}$, with similarly for the sub-vector $\eta_{V_{1}}$. Let $G=H^{-1}$. Notice $H>0$, a.s. and it is an $M$ matrix (see [PB74]). Therefore the entries of $G$ are all positive, a.s.. From the proof of Theorem A, we deduce the following.

Proposition 3.4. Let $\beta$ be a random variable with distribution $\nu^{W, \theta, \eta}$. Let $V_{1}=V \backslash\left\{i_{0}\right\}$ and $V_{2}=\left\{i_{0}\right\}$. The density of the marginal $\left(\beta_{i}\right)_{i \in V_{1}}$ is

$$
\left(\frac{2}{\pi}\right)^{\left|V_{1}\right| / 2} e^{-\frac{1}{2}\left(\left\langle\theta_{V_{1}}, H_{V_{1}, V_{1}} \theta_{V_{1}}\right\rangle+\left\langle\hat{\eta}_{V_{1}}, \hat{G}^{V_{1}} \hat{\eta}_{V_{1}}\right\rangle-2\left\langle\theta_{V_{1}}, \hat{\eta}_{V_{1}}\right\rangle\right)} \frac{\mathbb{1}_{H_{V_{1}, V_{1}}>0}}{\sqrt{\operatorname{det} H_{V_{1}, V_{1}}}} \prod_{i \in V_{1}} \theta_{i} d \beta_{i}
$$

where $\hat{\eta}_{V_{1}}=\eta_{V_{1}}+W_{V_{1}, V \backslash V_{1}} \theta_{V \backslash V_{1}}$ and $\hat{G}^{V_{1}}=\left(H_{V_{1}, V_{1}}\right)^{-1}$. The random variable $\beta_{i_{0}}$ can be represented as

$$
\beta_{i_{0}}=\gamma+\frac{1}{2}\left\langle W_{i_{0}, \cdot}, \hat{G}^{V_{1}} W_{i_{0}, \cdot}\right\rangle,
$$

where the density of $\gamma$, conditionally to $\left(\beta_{i}\right)_{i \in V_{1}}$, equals to

$$
\mathbb{1}_{\gamma>0} \theta_{i_{0}} \frac{1}{\sqrt{\pi}} e^{-\theta_{i_{0}}^{2} \gamma-\frac{1}{4 \gamma} \check{\eta}_{i_{0}}^{2}+\theta_{i_{0}} \check{\eta}_{i_{0}}} \frac{1}{\sqrt{\gamma}} d \gamma
$$


where

$$
\check{\eta}_{i_{0}}=\sum_{j \sim i_{0}} \sum_{k \in V_{1}} W_{i_{0}, j} \hat{G}^{V_{1}}(j, k) \eta_{k}+\eta_{i_{0}}
$$

Moreover,

$$
\gamma=\beta_{i_{0}}-\frac{1}{2}\left\langle W_{i_{0}, \cdot}, \hat{G}^{V_{1}} W_{i_{0}, \cdot}\right\rangle=\beta_{i_{0}}-\frac{1}{2} \sum_{j: j \sim i_{0}} W_{i_{0}, j} \frac{G\left(i_{0}, j\right)}{G\left(i_{0}, i_{0}\right)}=\frac{1}{2 G\left(i_{0}, i_{0}\right)},
$$

and $\gamma$ is independent of $\left(\beta_{i}\right)_{i \in V_{1}}$. In particular, if $\eta \equiv 0$ then $\gamma$ is Gamma $\left(\frac{1}{2}, \frac{1}{\theta_{i_{0}}^{2}}\right)$ distributed, with mean $\theta_{i_{0}}^{2} / 2$.

Proof. This is a corollary of the proof of Theorem A, given in the Appendix. It is a direct application of the fact that the density factorize into product of (7.5) and (7.6). Equation (3.6) follows from definition of Green function, that is, for any $i \in V$, we have

$$
2 \beta_{i} G\left(i_{0}, i\right)-\sum_{j: j \sim i} W_{i, j} G\left(i_{0}, j\right)=\mathbb{1}_{i=i_{0}} .
$$

Notice that by considering elements $j \in V_{1}$ in (3.7), one gets

$$
\hat{G}^{V_{1}} W_{i_{0}, \cdot}=H^{-1} W_{i_{0}, \cdot}=\frac{G\left(i_{0}, \cdot\right)}{G\left(i_{0}, i_{0}\right)} .
$$

The measures $\nu^{W, \theta, \eta}$ satisfy useful properties, or more specifically they enjoy some symmetries. For example, we can differentiate with respect to the parameters and obtain useful identities. Identities of this kind are called Ward identities, we list two of them, which will be useful later. For other Ward identities, one can check, for example, [DMR17a, DMR17b, DSZ10, SZ19, MRT16, BHS18]. Recall that we denote by $\langle\cdot, \cdot\rangle$ the usual inner product for vectors in $\mathbb{R}^{d}$. Moreover, for any continuous function $g: \mathbb{R}^{d} \mapsto \mathbb{R}^{+}$, we define

$$
\langle g(\beta)\rangle_{W, \theta, \eta}:=\int g(\beta) \nu^{W, \theta, \eta}(d \beta) .
$$

Remark 3.5. For Proposition 3.4, it is possible to prove that $\gamma$ is Gamma distributed via Laplace transform. Assume that $\beta$ is $\nu^{W, \theta, 0}$ distributed. Let $k>0$, define $\left(\eta_{i}\right)_{i \in V}$ by $\eta_{i_{0}}=$ $k$ and $\eta_{i}=0$ for $i \neq i_{0}$, by (3.2), we see that the Laplace transform of $\frac{1}{2 \gamma}=H^{-1}\left(i_{0}, i_{0}\right)$ equals

$$
\left\langle e^{-\frac{1}{2} k^{2} H^{-1}\left(i_{0}, i_{0}\right)}\right\rangle_{W, \theta, 0}=e^{-k \theta_{i_{0}}} .
$$

This characterize the distribution of $\frac{1}{2 \gamma}$, and entails that $\gamma$ is Gamma distributed.

Corollary 3.6 (Ward identities). Consider a finite weighted graph $\mathcal{G}$, let $\beta$ be $\mu^{W, \theta, \eta}$ distributed. For any $|V|$-dimensional vector $k$, such that $k_{i} \geq 0$ for all $i \in V$, we have

$$
\left\langle e^{-\sum_{i \in V} k_{i} \beta_{i}}\right\rangle_{W, \theta, \eta}=\mathrm{e}^{-\left\langle\eta, \sqrt{k+\theta^{2}}-\theta\right\rangle-\sum_{\{i, j\} \in E} W_{i, j}\left(\sqrt{\left(\theta_{i}^{2}+k_{i}\right)\left(\theta_{i}^{2}+k_{j}\right)}-\theta_{i} \theta_{j}\right)} \prod_{i \in V} \frac{\theta_{i}}{\sqrt{\theta_{i}^{2}+k_{i}}} .
$$

Fix $l \neq i_{0}$, let $\Xi(k, W, \beta)=\frac{G\left(i_{0}, l\right)}{G\left(i_{0}, i_{0}\right)} \exp \left(\frac{k_{i_{0}}}{2 G\left(i_{0}, i_{0}\right)}-\sum_{i \in V} k_{i} \beta_{i}\right)$, then

$$
\langle\Xi(k, W, \beta)\rangle_{W, \theta, 0}=\frac{\prod_{i \neq i_{0}} \theta_{i}}{\prod_{i \neq l} \sqrt{k_{i}+\theta_{i}^{2}}} \exp \left(-\sum_{\{i, j\} \in E} W_{i, j}\left(\sqrt{\left(k_{i}+\theta_{i}^{2}\right)\left(k_{j}+\theta_{j}^{2}\right)}-\theta_{i} \theta_{j}\right)\right) .
$$

Proof. See Appendix. 


\section{The VRJP is a random walk in random environment}

Recall that we use $\mathbf{Y}=\left(Y_{t}\right)_{t \geq 0}$ to denote $\operatorname{VRJP}(\mathrm{W})$ defined in the introduction. In [ST15a], Sabot and Tarrès introduced the following time change to the VRJP:

$$
Z_{t}=Y_{D^{-1}(t)}, \quad \text { with } t \in[0, \infty),
$$

where $D(s)$ is the following increasing random time change

$$
D(s)=\sum_{i \in V}\left(L_{i}^{2}(s)-\theta_{i}^{2}\right)=\sum_{i \in V} S_{i}(s),
$$

in particular, $S_{i}(s)=\int_{0}^{s} \mathbb{1}_{Z_{u}=i} d u$ is the local time of $\mathbf{Z}=\left(Z_{t}\right)$. This section is devoted to the study of $\mathbf{Z}$, which turns out to be a mixture of Markov jump processes. By definition, conditionally on $\left\{Z_{s}, s \leq t, Z_{t}=i\right\}$, at time $t, \mathbf{Z}$ jumps from $i$ to $j$ at rate

$$
W_{i, j} L_{j}(t) d L_{i}(t)=W_{i, j} \sqrt{S_{j}(t)+\theta_{j}^{2}} d \sqrt{S_{i}(t)+\theta_{i}^{2}}=\frac{1}{2} W_{i, j} \sqrt{\frac{S_{j}(t)+\theta_{j}^{2}}{S_{i}(t)+\theta_{i}^{2}}} d S_{i}(t) .
$$

The following theorem is first proved in [ST15a], the short proof we provided here is inspired by [ST15b, STZ17] and [Zen16], it is written in the context of the measure $\nu^{W, \theta, \eta}$, to provide a self-contained treatise of recurrence of the ERRW.

Theorem B. Consider a finite weighted graph $\mathcal{G}=(V, E, W, \theta)$. Let $\mathbf{Z}$ be the time changed VRJP starting from $i_{0} \in V$ defined by (4.1). We have that $\mathbf{Z}$ is a mixture of Markov jump processes.

More precisely, if $H=H_{W, \theta, 0}$ with $\theta>0$, is the random operator associated to $\mathcal{G}$ and introduced in Definition 3.3. Denote by $\mathbf{P}$ the distribution of $G=H^{-1}$. Define, for any collection of positive real numbers $\left(g\left(i_{0}, j\right)\right)_{j \in V}$, a Markov jump process $\left(\mathbf{X}, P^{g}\right)$. For which under the measure $P^{g}$ (called the quenched measure) the process $\mathbf{X}$ has Markov jump rates $\frac{1}{2} W_{i, j} \frac{g\left(i_{0}, j\right)}{g\left(i_{0}, i\right)}$ from $i$ to $j$. Then the annealed law of $\mathbf{X}$, defined by

$$
\mathbb{P}(\cdot)=\int P^{g}(\cdot) \mathbf{P}(d g)
$$

equals to the law of $\mathbf{Z}$. Also denote by $\mathbb{E}$ the associated expectation.

Proof. By (3.7), under the quenched measure $P^{g}$, the process $\mathbf{X}$ has sojourn time rate at $i \in V$ equal to

$$
\sum_{j: j \sim i} \frac{1}{2} W_{i, j} \frac{g\left(i_{0}, j\right)}{g\left(i_{0}, i\right)}=\left\{\begin{array}{ll}
\beta_{i} & i \neq i_{0} \\
\beta_{i_{0}}-\frac{1}{2 g\left(i_{0}, i_{0}\right)} & i=i_{0}
\end{array},\right.
$$

Given a trajectory

$$
\sigma: X_{\left[0, t_{1}\right)}=i_{0}, X_{\left[t_{1}, t_{2}\right)}=i_{1}, \cdots, X_{\left[t_{n-1}, t_{n}\right)}=i_{n-1}, X_{\left[t_{n}, t\right)}=i_{n},
$$

let $S_{i}(t)$ be the local time of $\mathbf{X}$ up to time $t$. The quenched trajectory density (see Definition 4 of [Zen16]) of $\mathbf{X}$ equals

$$
f_{\text {quenched }}^{X}(\sigma)=\prod_{k=1}^{n}\left(\frac{1}{2} W_{i_{k-1}, i_{k}}\right) \frac{g\left(i_{0}, i_{n}\right)}{g\left(i_{0}, i_{0}\right)} \exp \left(-\sum_{i \in V} S_{i}(t) \beta_{i}+\frac{S_{i_{0}}(t)}{2 g\left(i_{0}, i_{0}\right)}\right) .
$$

By the Ward identity (3.10), the annealed trajectory density of $\mathbf{X}$ is

$$
\begin{aligned}
f_{\text {annealed }}^{X}(\sigma)= & \prod_{k=1}^{n}\left(\frac{1}{2} W_{i_{k-1}, i_{k}}\right) \frac{\prod_{i: i \neq i_{0}} \theta_{i}}{\prod_{i: i \neq i_{n}} \sqrt{S_{i}(t)+\theta_{i}^{2}}} \\
& \times \exp \left(-\sum_{i, j: i \sim j} W_{i, j}\left(\sqrt{\left(S_{i}(t)+\theta_{i}^{2}\right)\left(S_{j}(t)+\theta_{j}^{2}\right)}-\theta_{i} \theta_{j}\right)\right) .
\end{aligned}
$$


On the other hand, the trajectory density of $\mathbf{Z}$ equals (we abuse notation and denote by $S_{i}(t)$ the local time of $\mathbf{Z}$, note also $S_{i_{k}}\left(t_{k}\right)=S_{i_{k}}\left(t_{k-1}\right)$ )

$$
\begin{aligned}
f^{Z}(\sigma) & =\prod_{k=1}^{n}\left(\frac{1}{2} W_{i_{k-1}, i_{k}} \sqrt{\frac{S_{i_{k}}\left(t_{k-1}\right)+\theta_{i_{k}}^{2}}{S_{i_{k-1}}\left(t_{k}\right)+\theta_{i_{k-1}}^{2}}}\right) \cdot \exp \left(-\int_{0}^{t} \sum_{j: j \sim Z_{v}} \frac{1}{2} W_{Z_{v}, j} \sqrt{\frac{S_{j}(v)+\theta_{j}^{2}}{S_{Z_{v}}(v)+\theta_{Z_{v}}^{2}}} d v\right) \\
& =\prod_{k=1}^{n}\left(\frac{1}{2} W_{i_{k-1}, i_{k}}\right) \frac{\prod_{i \neq i_{0}} \theta_{i}}{\prod_{i \neq i_{n}} \sqrt{S_{i}(t)+\theta_{i}^{2}}} \cdot \exp \left(-\int_{0}^{t} \sum_{j: j \sim Z_{v}} \frac{1}{2} W_{Z_{v}, j} \sqrt{\frac{S_{j}(v)+\theta_{j}^{2}}{S_{Z_{v}}(v)+\theta_{Z_{v}}^{2}}} d v\right) .
\end{aligned}
$$

Now since

$$
\frac{d}{d t}\left(\sum_{i, j: i \sim j} W_{i, j}\left(\sqrt{\left(S_{i}(t)+\theta_{i}^{2}\right)\left(S_{j}(t)+\theta_{j}^{2}\right)}-\theta_{i} \theta_{j}\right)\right)=\sum_{j: j \sim Z_{t}} \frac{1}{2} W_{Z_{t}, j} \sqrt{\frac{S_{j}(t)+\theta_{j}^{2}}{S_{Z_{t}}(t)+\theta_{Z_{t}}^{2}}},
$$

we conclude that $f^{Z}(\sigma)=f_{\text {annealed }}^{X}(\sigma)$, hence the law of $\mathbf{X}$ and $\mathbf{Z}$ are equal.

The following corollary is immediate.

Corollary 4.1. Fix a finite weighted graph $\mathcal{G}$. Let $H_{\beta}$ the random operator associated to $\mathcal{G}$ (as per Definition 3.3) and $G=H_{\beta}^{-1}$. The discrete time process $\left(Y_{n}\right)$ associated to the VRJP is a random conductance model, where the conductance on the edge $\{i, j\} \in E$ is $C_{i, j}=W_{i, j} G\left(i_{0}, i\right) G\left(i_{0}, j\right)$.

\section{Recurrence with strong reinforcement on $\mathbb{Z}^{d}$}

We can actually show that the random potential $\beta$ can be defined on a infinite graph, see Proposition 1 of [SZ19] for the case $\eta \equiv 0$.

Theorem C. One can extend to $\nu^{W, \theta, \eta}$ to the entire $\mathbb{Z}^{d}$. If $\left(\beta_{i}\right)_{i \in \mathbb{Z}^{d}}$ is $\nu^{W, \theta, \eta}$ distributed, its law is characterized by the Laplace transform of its finite dimensional marginals: for any finite collection of vertices $V \subset \mathbb{Z}^{d}$,

$$
\begin{aligned}
\left\langle\mathrm{e}^{-\sum_{i \in V} k_{i} \beta_{i}}\right\rangle_{W, \theta, \eta}= & \mathrm{e}^{-\sum_{i \in V} \eta_{i}\left(\sqrt{k_{i}+\theta_{i}^{2}}-\theta_{i}\right)-\sum_{\{i, j\} \in E\left(\mathbb{Z}^{d}\right)} W_{i, j}\left(\sqrt{\left(\theta_{i}^{2}+k_{i}\right)\left(\theta_{j}^{2}+k_{j}\right)}-\theta_{i} \theta_{j}\right)} \\
& \times \prod_{i \in V} \frac{\theta_{i}}{\sqrt{\theta_{i}^{2}+k_{i}}},
\end{aligned}
$$

where $\left(k_{i}\right)_{i \in V}$ is such that $k_{i} \geq 0$, and conventionally $k_{i}=0$ if $i \notin V$, in particular, the $\operatorname{sum} \sum_{\{i, j\} \in E\left(\mathbb{Z}^{d}\right)}$ in the exponent is a finite sum.

Proof. This is a direct application of the Kolmogorov extension theorem, and the fact that by (3.9), $\beta_{i}$ and $\beta_{j}$ are independent if $i \nsim j$.

Remark 5.1. In particular, if $\beta$ is $\nu^{W, \theta, 0}$ distributed, then $\theta^{2} \beta=\left(\theta_{i}^{2} \beta_{i}\right)_{i \in V}$ is $\nu^{W^{\theta}, 1,0}$ distributed, where $W_{i, j}^{\theta}=W_{i, j} \theta_{i} \theta_{j}$. Moreover, in the case where $W$ and $\theta$ are constant, if $\beta$ is $\nu^{W, 1,0}$ distributed, then $\beta / W$ is $\nu^{1, \sqrt{W}, 0}$ distributed.

In the sequel, we focus on the graph $\mathbb{Z}^{d}$. In order to have homogeneity, we assume that $\theta_{i}$ and $W_{i, j}$ are constant, equals to $\theta$ and $W$ respectively. Define the random operator associated to $\mathbb{Z}^{d}$, by Definition 3.1, it is of the form $H_{\beta}=2[\beta]-\Delta_{W}$, where $\beta$ is $\nu^{W, \theta, 0}$ distributed, as in Theorem C. By setting $\beta_{j}=0, \forall j \neq i$ in (5.1), we see that $1 /\left(2 \beta_{i}\right)$ is an IG $\left(\left(\sum_{j: j \sim i} W_{i, j} \theta_{j} / \theta_{i}\right)^{-1}, \theta_{i}^{2}\right)$ distributed random variable, in particular, the variance of $\beta_{i}$ is (since $W_{i, j}$ and $\theta_{i}$ are constant):

$$
\left\langle\beta_{i}^{2}\right\rangle_{W, \theta, 0}-\left\langle\beta_{i}\right\rangle_{W, \theta, 0}^{2}=\frac{1}{2 \theta_{i}^{2}}+\frac{\sum_{j} W_{i, j} \theta_{j}}{4 \theta_{i}^{3}}=\frac{1+W d}{2 \theta^{2}} .
$$


To conjugate our language to the one that is usually used in the context of random Schrödinger operator, we have to normalize $H_{\beta}$ in such a way that the kinetic energy is associated to the unweighted Laplacian $\Delta$ (that is, with entry 1 or 0 depending whether an edge is present), so we would rather consider $H_{\beta} / W$ instead, and then the coupling constant is the variance of the potential $2 \beta / W$, that is

$$
\frac{2}{\theta^{2} W^{2}}+\frac{2 d}{\theta^{2} W} \text {. }
$$

In particular, either $\theta$ small or $W$ small will entail large variance, i.e. strong disorder, and we would expect localization.

For a finite box $\Lambda$ of $\mathbb{Z}^{d}$, consider the finite weighted graph induced by the wired boundary condition, denoted $\widetilde{\Lambda}$, and defined as follows. Let $\Lambda=(V, E, W, \theta)$. The set of vertices of $\widetilde{\Lambda}$, denoted by $V \cup\{\delta\}$ where $\delta$ is an additional vertex. The graph $\widetilde{\Lambda}$ is obtained from $\Lambda$ by adding edges connecting $\delta$ to each of the vertices on the boundary, which in turn is defined as $\{i: \exists j \sim i, j \notin V\}$. Moreover

$$
W_{\delta, i}=\sum_{j: j \sim i, j \notin V} W_{i, j}
$$

and $\theta_{\delta}=\theta$.

Lemma 5.2. For any finite graph $\mathcal{G}=(V, E)$, in particular, finite boxes of $\mathbb{Z}^{d}$, if $H=$ $H_{W, \theta, 0}$ is the associated operator, with $\eta \equiv 0$. Then, for any $i_{0} \in V$

$$
\left\langle H^{-1}\left(i_{0}, i_{0}\right)^{1 / 4}\right\rangle_{W, \theta, 0} \leq \frac{\Gamma(1 / 4)}{2^{1 / 4} \sqrt{\pi}} \sqrt{\theta} .
$$

Note that this bound is completely independent of $W$.

Proof. We recall that $H>0$ which implies $H^{-1}\left(i_{0}, i_{0}\right)>0$. By Corollary $3.4,1 /\left(2 H^{-1}\left(i_{0}\right.\right.$, $\left.\left.i_{0}\right)\right)$ is a Gamma variable with parameters $\left(\frac{1}{2}, \frac{1}{\theta^{2}}\right)$, Hence

$$
\begin{aligned}
\left\langle\left(2 H^{-1}\left(i_{0}, i_{0}\right)\right)^{1 / 4}\right\rangle_{W, \theta, 0} & =\left\langle\left(\frac{1}{2 H^{-1}\left(i_{0}, i_{0}\right)}\right)^{-1 / 4}\right\rangle_{W, \theta, 0} \\
& =\int_{0}^{\infty} u^{-1 / 4} \frac{u^{-1 / 2}}{\Gamma(1 / 2)} \frac{1}{\theta} \mathrm{e}^{-u / \theta^{2}} \mathrm{~d} u \\
& =\frac{\Gamma(1 / 4)}{\sqrt{\pi}} \sqrt{\theta} .
\end{aligned}
$$

From now on, we denote by $x \rightarrow y$ the collection of paths connecting $x$ to $y$ for any pair of vertices $x$ and $y$. Moreover, for any connected set $A \subset V$, containing $x, y$, denote by $(x \rightarrow y, A)$ the collection of paths connecting $x$ to $y$ and whose vertices belong to $A$ only. For any path $\sigma$, we denote by $|\sigma|$ its length.

Proposition 5.3 (Random walk expansion). Let $H_{\beta}$ be a random Schrödinger operator on a finite graph, and let $G$ be the relative Green function, i.e. $G=H_{\beta}^{-1}$. Let $\sigma: x \rightarrow y$ be a finite admissible path connecting $x, y \in V$, that is, $\sigma=\left(\sigma_{1}=x, \sigma_{2}, \ldots, \sigma_{|\sigma|}=y\right)$, define

$$
W_{\sigma}=\prod_{k=1}^{|\sigma|-1} W_{\sigma_{k}, \sigma_{k+1}}, \quad(2 \beta)_{\sigma}=\prod_{k=1}^{|\sigma|}\left(2 \beta_{\sigma_{k}}\right),
$$

then we have

$$
G(x, y)=\sum_{\sigma \in x \rightarrow y} \frac{W_{\sigma}}{(2 \beta)_{\sigma}} .
$$



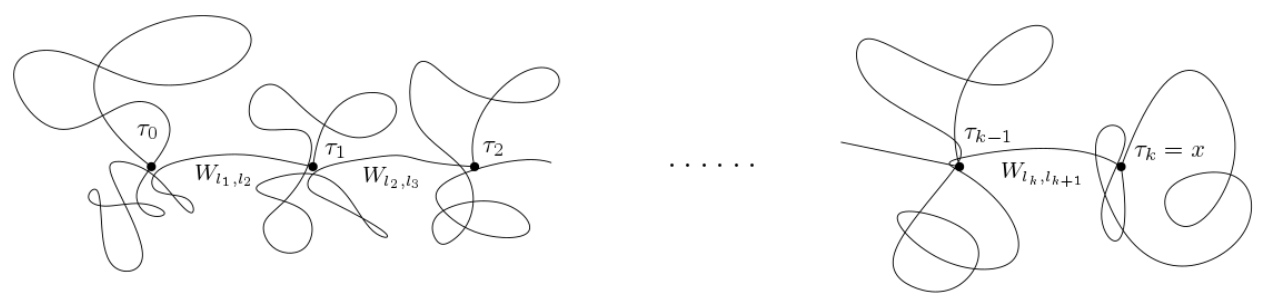

Figure 1: A path is decomposed into its loop-erased path along with dangling cycles.

Proof. Let $D$ be the diagonal matrix with coefficient $2 \beta_{i}$, we have

$$
D^{-1 / 2} H D^{-1 / 2}=I-D^{-1 / 2} \Delta_{W} D^{-1 / 2}:=I-A .
$$

Since $H>0$ a.s., we have, for any vector $\varphi \in \mathbb{R}^{V}$, since $D^{1 / 2}$ is self-adjoint,

$$
\langle H \varphi, \varphi\rangle=\left\langle D^{1 / 2}(I-A) D^{1 / 2} \varphi, \varphi\right\rangle=\left\langle(I-A) D^{1 / 2} \varphi, D^{1 / 2} \varphi\right\rangle>0 .
$$

Let $\psi=D^{1 / 2} \varphi$, we have $\|\psi\|^{2}>\langle A \psi, \psi\rangle$ for all $\psi$, therefore, the largest real positive eigenvalue of $A$ is less than one. Recall that a.s. $\beta_{i}>0$ for all $i$; by definition of $A$ and the fact that we consider a connected graph, we have that a.s. $A$ is an irreducible matrix with non-negative coefficients. By the Perron-Frobenius theorem, the largest eigenvalue in absolute value, i.e. the Perron root of $A$, denoted by $r(A)$, is real and positive. From the reasoning above, we have that $r(A)<1$. As a consequence, $\|A\|_{o p}<1$. Next, we write

$$
\begin{aligned}
G & =H^{-1}=D^{-1 / 2}(I-A)^{-1} D^{-1 / 2} \\
& =D^{-1 / 2}\left(\sum_{k=0}^{\infty} A^{k}\right) D^{-1 / 2}=D^{-1} \sum_{k=0}^{\infty}\left(\Delta_{W} D^{-1}\right)^{k} .
\end{aligned}
$$

The above series convergence holds in operator norm, in particular, also holds in the weak operator topology, which is the random walk expansion.

Proof of Theorem 2.1 part 1). By Remark 5.1, it suffice to deal with the case $\theta=1$ and $\left(\widetilde{W}_{e}\right)_{e \in E}$ random and independent. Set

$$
W^{\prime}=\sup _{e} \mathbb{E}\left[\widetilde{W}_{e}^{1 / 4}\right]<\bar{W}_{d}
$$

We will combine the random walk expansion and the fact that diagonal Green function is a Gamma variable (which's distribution is independent of the graph $\mathcal{G}$, and, as a consequence, all the bounds below are uniform on the sub-graphs on which the self avoiding paths live, except that the number of such paths depend on the graph). Notice that the collection of paths $\sigma: 0 \rightarrow x$, can be decomposed as follows.

As shown in Figure 1, the collection of paths from $x$ to $y$, can be seen as the collection of self-avoiding paths from $x$ to $y$ (denoted by $\tau$ in the figure) along with the collection of paths from $\tau_{i}$ to $\tau_{i}$ for all $i$ (which are the loops).

We can therefore factorize the random walk expansion according to this path cut, 
and write

$$
\begin{aligned}
& G(0, x)=\sum_{\sigma \in 0 \rightarrow x} \frac{\widetilde{W}_{\sigma}}{(2 \beta)_{\sigma}} \\
& =\sum_{\tau \in 0 \Rightarrow x}\left(\sum_{\pi_{0} \in 0 \rightarrow 0} \frac{\widetilde{W}_{\pi_{0}}}{(2 \beta)_{\pi_{0}}}\right) \widetilde{W}_{l_{1}, l_{2}}\left(\sum_{\pi_{1} \in\left(\tau_{1} \rightarrow \tau_{1}, V \backslash\left\{\tau_{0}\right\}\right)} \frac{\widetilde{W}_{\pi_{1}}}{(2 \beta)_{\pi_{1}}}\right) \widetilde{W}_{l_{2}, l_{3}} \ldots \widetilde{W}_{l_{k}, l_{k+1}} \\
& \left(\sum_{\pi_{k} \in\left(\tau_{k} \rightarrow \tau_{k}, V \backslash\left\{\tau_{0}, \ldots, \tau_{k-1}\right\}\right)} \frac{\widetilde{W}_{\pi_{k}}}{(2 \beta)_{\pi_{k}}}\right)
\end{aligned}
$$

Notice that $\left(\sum_{\pi_{0} \in 0 \rightarrow 0} \frac{\widetilde{W} v_{\pi_{1}}}{(2 \beta)_{\pi_{1}}}\right)=G(0,0)$, it is distributed like the reciprocal of a Gamma random variable, and it is independent of the rest. Unfortunately, the reciprocal of Gamma random variable do not have first moment, thus we compute a fractional moment instead, and use the following bound which holds for any $a_{1}, \ldots, a_{n}>0$,

$$
\left(\sum_{i} a_{i}\right)^{1 / 4} \leq \sum_{i} a_{i}^{1 / 4}
$$

Hence, if we denote $C=\mathbb{E}\left(G(0,0)^{1 / 4}\right)$, which is a constant, and independent of $\left(\widetilde{W}_{i, j}\right)$ by Lemma 5.2. We have

$$
\begin{aligned}
\mathbb{E}\left[G(0, x)^{1 / 4}\right] \leq & \sum_{\tau: 0 \Rightarrow x} \mathbb{E}\left[\left(G(0,0) \widetilde{W}_{l_{1}, l_{2}}\left(\sum_{\pi_{1}: \tau_{1} \rightarrow \tau_{1} \in V \backslash\left\{\tau_{0}\right\}} \frac{\widetilde{W}_{\pi_{1}}}{(2 \beta)_{\pi_{1}}}\right) \widetilde{W}_{l_{2}, l_{3}} \widetilde{W}_{l_{k}, l_{k+1}}\right.\right. \\
& \left.\left.\times\left(\sum_{\pi_{k}: \tau_{k} \rightarrow \tau_{k} \in V \backslash\left\{\tau_{0}, \ldots, \tau_{k-1}\right\}} \frac{\widetilde{W}_{\pi_{k}}}{(2 \beta)_{\pi_{k}}}\right)\right)^{1 / 4}\right] \\
= & W^{\prime} C \sum_{\tau: 0 \Rightarrow x} \mathbb{E}\left[\left(\sum_{\pi_{1}: \tau_{1} \rightarrow \tau_{1} \in V \backslash\left\{\tau_{0}\right\}} \frac{\widetilde{W}_{\pi_{1}}}{(2 \beta)_{\pi_{1}}}\right) \widetilde{W}_{l_{2}, l_{3}} \ldots \widetilde{W}_{l_{k}, l_{k+1}}\right. \\
& \left.\left.\times\left(\sum_{\pi_{k}: \tau_{k} \rightarrow \tau_{k} \in V \backslash\left\{\tau_{0}, \ldots, \tau_{k-1}\right\}} \frac{\widetilde{W}_{\pi_{k}}}{(2 \beta)_{\pi_{k}}}\right)\right)^{1 / 4}\right]
\end{aligned}
$$

Notice that

$$
\left(\sum_{\pi_{1}: \tau_{1} \rightarrow \tau_{1} \in V \backslash\left\{\tau_{0}\right\}} \frac{\widetilde{W}_{\pi_{1}}}{(2 \beta)_{\pi_{1}}}\right) \leq \sum_{\pi: \tau_{1} \rightarrow \tau_{1}} \frac{\widetilde{W}_{\pi}}{(2 \beta)_{\pi}}=G\left(\tau_{1}, \tau_{1}\right)
$$

and the rest inside the expectation is independent of $G\left(\tau_{1}, \tau_{1}\right)$. Hence continuing our inequality we have

$$
\begin{aligned}
\mathbb{E}\left[G(0, x)^{1 / 4}\right] & \leq\left(W^{\prime} C\right)^{2} \sum_{\tau: 0 \Rightarrow x} \mathbb{E}\left[\left(\sum_{\pi_{2}: \tau_{2} \rightarrow \tau_{2} \in V \backslash\left\{\tau_{0}, \tau_{1}\right\}} \frac{\widetilde{W}_{\pi_{2}}}{(2 \beta)_{\pi_{2}}}\right) \widetilde{W}_{l_{3}, l_{4}} \ldots\right. \\
& \left.\left.\widetilde{W}_{l_{k}, l_{k+1}}\left(\sum_{\pi_{k}: \tau_{k} \rightarrow \tau_{k} \in V \backslash\left\{\tau_{0}, \ldots, \tau_{k-1}\right\}} \frac{\widetilde{W}_{\pi_{k}}}{(2 \beta)_{\pi_{k}}}\right)\right)^{1 / 4}\right],
\end{aligned}
$$


recursively we get, at the end

$$
\mathbb{E}\left[G(0, x)^{1 / 4}\right] \leq \sum_{\tau: 0 \Rightarrow x}\left(W^{\prime} C\right)^{|\tau|} \leq \sum_{k \geq|x| \tau: 0 \Rightarrow x,|\tau|=k} \sum_{k \geq|x|}\left(W^{\prime} C\right)^{k} \leq \sum_{k}(2 d)^{k}\left(W^{\prime} C\right)^{k} \leq e^{-\kappa|x|}
$$

where we have used the fact that there is at most $(2 d)^{k}$ self avoiding paths on a finite sub graph of $\mathbb{Z}^{d}$ of length $k$, and have chosen $W^{\prime}$ such that $(2 d)\left(W^{\prime} C\right)<1$.

Finally, either using a tightness argument as in [MR09], or applying directly Theorem 1.(iii) in [SZ19], VRJP and ERRW are random walk in random conductance, and (5.4) implies exponential decay of the conductance, therefore, a.s. sum of the conductance are finite, which in turn implies that the process is positively recurrent.

\section{Pure point spectrum: proof Theorem 2.4}

Since $\beta=\left(\beta_{i}\right)_{i \in V\left(\mathbb{Z}^{d}\right)}$ be $\nu^{1, \theta, 0}$ distributed, by the discussion after Remark 5.1, $H_{\theta}$ is in the right scaling. We use a result of [AM93], in particular, we recall the following definition:

Definition 6.1. A probability measure $\nu$ on $\mathbb{R}$ is said to be $\tau$-regular for some parameter $\tau \in(0,1)$ if there exists finite constants $C, K>0$ such that

$$
\nu([z-\delta, z+\delta]) \leq C \delta^{\tau} \nu([z-K, z+K])
$$

for all $\delta \in(0,1)$ and $z \in \mathbb{R}$.

According to Theorem 3.1 of [AM93] it suffices for our purpose to show that, the conditional density of single site potential is $\tau$-regular for some $\tau>0$. Let $\mathcal{G}_{n}=\left(V_{n}, E_{n}\right)$ be the cube of volume $2^{d} n^{d}$ centered at the origin. Denote by $\partial V_{n}$ the boundary of this cube, i.e. the set of vertices $y \notin V_{n}$ which are neighbors to at least one element of $V_{n}$. By (5.1), the marginal distribution $\left(\beta_{i}\right)_{i \in V}$ is $\nu^{1, \theta, \eta}$ distributed, where (note that $W \equiv 1$ )

$$
\eta_{i}=\sum_{j: j \sim i, j \in \partial V_{n}} \theta=\theta \cdot \operatorname{card}\left\{j: j \sim i, j \in \partial V_{n}\right\}=O\left(n^{d-1}\right) .
$$

By Remark 5.1 and the proof of Theorem 2.1 part 1), we can choose $\theta_{0}$ such that if we consider $\operatorname{VRJP}(1, \theta)$, with $\theta<\theta_{0}$, then

$$
\mathbb{E}\left[\left(\widehat{G}^{V_{n}}(0, x)\right)^{1 / 4}\right] \leq \mathrm{e}^{-\kappa|x|},
$$

for some $\kappa>0$. In the sequel assume $\theta<\theta_{0}$. The conditional density of $\beta_{0}$ given $\left(\beta_{i}\right)_{i \in V \backslash\{0\}}$ is given by (3.4), with $i_{0}=0$. Using (3.5) and similar argument as in (5.3) that, (6.1) holds with $j, k$ instead of $0, x$, we have, a.s.

$$
\check{\eta}_{0}=\sum_{j: j \sim 0} \sum_{k \in V_{n}} \hat{G}^{V_{n} \backslash\{0\}}(j, k) \eta_{k} \leq O\left(n^{d-1}\right) e^{-\kappa n} \stackrel{n \rightarrow \infty}{\longrightarrow} 0 .
$$

Note that, recall the definition of $\eta_{k}$, in the above sum, only those $k$ s.t. that is connected to at least one vertex outside $V_{n}$ are non zero, and the number of such $k$ is $O\left(n^{d-1}\right)$.

Define

$$
D_{0}=\lim _{n \rightarrow+\infty} \sum_{j: j \sim i_{0}} \frac{G^{V_{n}}\left(i_{0}, j\right)}{G^{V_{n}}\left(i_{0}, i_{0}\right)}
$$

Note that by random walk expansion, $\frac{G^{V_{n}}\left(i_{0}, j\right)}{G^{V_{n}}\left(i_{0}, i_{0}\right)}$ is the sum over weight on paths in $V_{n}$, start from $i_{0}$ that touch $j$ without returning to $i_{0}$, this is an increasing function of $V_{n}$, as 
the path weights are positive. In particular, there is no problem in exchanging limit and expectation. Notice that $D_{0}<\infty$ a.s. In fact, using Cauchy-Schwartz

$$
\mathbb{E}\left[\left(\frac{G\left(i_{0}, j\right)}{G\left(i_{0}, i_{0}\right)}\right)^{1 / 8}\right] \leq \mathbb{E}\left[G\left(i_{0}, j\right)^{1 / 4}\right]^{1 / 2} \mathbb{E}\left[G\left(i_{0}, i_{0}\right)^{-1 / 4}\right]^{1 / 2}<\infty .
$$

The finiteness of the expression above is a consequence of (6.1) and the fact that $\gamma$ (see equation (3.6)) is Gamma distributed with parameter independent of the sub graph $V_{n}$. It follows that, by Proposition 3.4, and taking $V \uparrow \mathbb{Z}^{d}$, the density of $\beta_{0}$ conditioned on $\left(\beta_{i}\right)_{i \in V\left(\mathbb{Z}^{d}\right) \backslash\{0\}}$ equals

$$
g_{D_{0}}(u):=\frac{1}{\sqrt{2 \pi}} \exp \left(-\left(u-D_{0}\right) \theta^{2} / 2\right) \frac{\theta}{\sqrt{u-D_{0}}} \mathbb{1}_{u \geq D_{0}} d u .
$$

We show that $g_{D_{0}}(u) d u$ is $\frac{1}{2}$-regular (c.f. p256 of [AM93]), note that, $g_{D_{0}}$ vanish in $\left(-\infty, D_{0}\right)$, and $\lim _{u \downarrow D_{0}} g_{D_{0}}(u)=\infty$, and $g_{D_{0}}$ is decreasing in $\left(D_{0}, \infty\right)$. Hence $g_{D_{0}}$ is $\frac{1}{2}$-regular (thanks to monotonicity and note that $0<\delta<1$ ) by setting $K=1, C=1$ in Definition 6.1, if $z$ is away from $D_{0}$ and $\delta$ is such that $z-\delta>D_{0}$. Therefore, it suffices to check Equation (3.1) of [AM93] at the singularity $D_{0}$, i.e., to check $\nu\left(\left[D_{0}, \delta\right]\right) \leq C \delta$. We can explicitly compute that,

$$
\int_{D_{0}}^{D_{0}+x} g_{D_{0}}(u) d u=\operatorname{Erf}(\theta \sqrt{x / 2})=\int_{0}^{\theta \sqrt{x / 2}} \frac{1}{\sqrt{2 \pi}} e^{-t^{2}} d t=\frac{1}{\sqrt{2 \pi}}\left(\theta \sqrt{x}-\frac{(\theta \sqrt{x})^{3}}{3}+o\left(x^{2}\right)\right) .
$$

Therefore, the conditional single site density (6.2) is $\frac{1}{2}$-regular. Thus, by Theorem 3.1 of [AM93], there exists a $\theta_{0}$, such that for $\theta<\theta_{0}$, the operator $H_{\theta}$ on $\mathbb{Z}^{d}$ is localized, which proves Theorem 2.4.

\section{Appendix A: Proof of Theorem A}

Proof. Recall that $V=\{1, \ldots, N\}$. We partition $V$ into $V=V_{1} \sqcup V_{2}$. After a relabelling, we can pick $V_{1}=\left\{1, \ldots, N^{\prime}\right\}$ and $V_{2}=\left\{N^{\prime}+1, \ldots, N\right\}$. The matrix $H$ can be written in block form

$$
H=\left(\begin{array}{cc}
H_{V_{1}, V_{1}} & -W_{V_{1}, V_{2}} \\
-W_{V_{2}, V_{1}} & H_{V_{2}, V_{2}}
\end{array}\right)
$$

where the top left block $H_{V_{1}, V_{1}}$ is an $N^{\prime} \times N^{\prime}$ matrix whose entries are $\left(H_{i, j}\right)_{i, j \in V_{1}}$. The other blocks are defined implicitly by the identity (7.1). The Schur decomposition of the right-hand side of (7.1) implies

$$
H=\left(\begin{array}{cc}
I_{V_{1}} & 0 \\
-W_{V_{2}, V_{1}} \hat{G}^{V_{1}} & I_{V_{2}}
\end{array}\right)\left(\begin{array}{cc}
H_{V_{1}, V_{1}} & 0 \\
0 & \check{H}^{V_{2}}
\end{array}\right)\left(\begin{array}{cc}
I_{V_{1}} & -\hat{G}^{V_{1}} W_{V_{1}, V_{2}} \\
0 & I_{V_{2}}
\end{array}\right)
$$

where $I_{V_{1}}$ (resp. $I_{V_{2}}$ ) is the identity matrix of dimension $N^{\prime}$ (resp. $N-N^{\prime}$ ). Moreover,

$$
\hat{G}^{V_{1}}=\left(H_{V_{1}, V_{1}}\right)^{-1}, \check{H}^{V_{2}}=H_{V_{2}, V_{2}}-W_{V_{2}, V_{1}} \hat{G}^{V_{1}} W_{V_{1}, V_{2}}, \check{G}^{V_{2}}=\left(\check{H}^{V_{2}}\right)^{-1} .
$$

The inverse of $H$ can be computed via this decomposition, that is

$$
G=H^{-1}=\left(\begin{array}{cc}
I_{V_{1}} & \hat{G}^{V_{1}} W_{V_{1}, V_{2}} \\
0 & I_{V_{2}}
\end{array}\right)\left(\begin{array}{cc}
\hat{G}^{V_{1}} & 0 \\
0 & \check{G}^{V_{2}}
\end{array}\right)\left(\begin{array}{cc}
I_{V_{1}} & 0 \\
W_{V_{2}, V_{1}} \hat{G}^{V_{1}} & I_{V_{2}}
\end{array}\right)
$$

Notice that when we take $V_{2}$ to be a singleton, the previous decomposition implies the second equality in (3.6). Note we can also write our vector $\theta, \eta$ in block form, that is, e.g. $\theta=\left(\theta_{V_{1}}, \theta_{V_{2}}\right)$. Recall that $\check{\eta}_{V_{2}}$ was defined via the equation

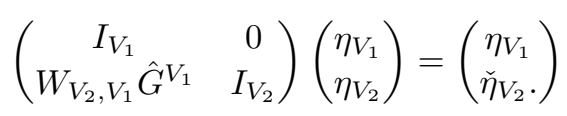


VRJP and fractional moment localization

and

$$
\hat{\eta}_{V_{1}}=\eta_{V_{1}}+W_{V_{1}, V_{2}} \theta_{V_{2}}
$$

With all these definitions and decompositions, the quadratic form in the exponent (which is the Hamiltonian or energy in the point of view of statistical physics) can be written as

$$
\begin{aligned}
\langle\theta, H \theta\rangle+\langle\eta, G \eta\rangle-2\langle\theta, \eta\rangle & =\left\langle\theta_{V_{2}}, \check{H}^{V_{2}} \theta_{V_{2}}\right\rangle+\left\langle\check{\eta}_{V_{2}}, \check{G}^{V_{2}} \check{\eta}_{V_{2}}\right\rangle-2\left\langle\check{\eta}_{V_{2}}, \theta_{V_{2}}\right\rangle \\
& +\left\langle\theta_{V_{1}}, H_{V_{1}, V_{1}} \theta_{V_{1}}\right\rangle+\left\langle\hat{\eta}_{V_{1}}, \hat{G}^{V_{1}} \hat{\eta}_{V_{1}}\right\rangle-2\left\langle\hat{\eta}_{V_{1}}, \theta_{V_{1}}\right\rangle .
\end{aligned}
$$

Note also that for the determinant, we have, again by Schur, $\operatorname{det} H=\operatorname{det} H_{V_{1}, V_{1}} \operatorname{det} \check{H}^{V_{2}}$, therefore, the integrand in Equation (3.2) factorizes

$$
\begin{aligned}
& \left(\frac{2}{\pi}\right)^{N / 2} e^{-\frac{1}{2}(\langle\theta, H \theta\rangle+\langle\eta, G \eta\rangle-2\langle\theta, \eta\rangle)} \frac{\mathbb{1}_{H>0}}{\sqrt{\operatorname{det} H}} \prod_{i} \theta_{i} d \beta \\
= & \left(\frac{2}{\pi}\right)^{\left|V_{1}\right| / 2} e^{-\frac{1}{2}\left(\left\langle\theta_{V_{1}}, H_{V_{1}, V_{1}} \theta_{V_{1}}\right\rangle+\left\langle\hat{\eta}_{V_{1}}, \hat{G}^{V_{1}} \hat{\eta}_{V_{1}}\right\rangle-2\left\langle\hat{\eta}_{V_{1}}, \theta_{V_{1}}\right\rangle\right)} \frac{\mathbb{1}_{H_{V_{1}, V_{1}}>0}}{\sqrt{\operatorname{det} H_{V_{1}, V_{1}}}} \prod_{i \in V_{1}} \theta_{i} d \beta_{V_{1}} \\
& \left(\frac{2}{\pi}\right)^{\left|V_{2}\right| / 2} e^{-\frac{1}{2}\left(\left\langle\theta_{V_{2}}, \check{H}^{V_{2}} \theta_{V_{2}}\right\rangle+\left\langle\check{\eta}_{V_{2}}, \check{G}^{V_{2}} \check{\eta}_{V_{2}}\right\rangle-2\left\langle\check{\eta}_{V_{2}}, \theta_{V_{2}}\right\rangle\right)} \frac{\mathbb{1}_{\check{H}^{V_{2}}>0}}{\sqrt{\operatorname{det} \check{H}^{V_{2}}}} \prod_{i \in V_{2}} \theta_{i} d \beta_{V_{2}}
\end{aligned}
$$

Next we choose $V_{2}=\left\{i_{1}\right\}$. Conditionally on $\beta_{V_{1}}$, and performing the change of variable $y=\beta_{i_{1}}-\left\langle W_{i_{1},}, \hat{G}^{V_{1}} W_{\cdot, i_{1}}\right\rangle$, we find the conditional density

$$
\frac{a}{\sqrt{2 \pi}} e^{-\frac{1}{2}\left(a^{2} y-b^{2} / y-2 a b\right)} \frac{1}{\sqrt{y}},
$$

for some $a, b>0$. In virtue of (3.3), this is a probability density. By iterating the previous step to each of the one-dimensional marginals, we prove Equation (3.2). Moreover, the factorization and the inductive argument used to integrate $\beta_{V_{2}}$ in (7.4) imply that

$$
\left(\frac{2}{\pi}\right)^{\left|V_{1}\right| / 2} e^{-\frac{1}{2}\left(\left\langle\theta_{V_{1}}, H_{V_{1}, V_{1}} \theta_{V_{1}}\right\rangle+\left\langle\hat{\eta}_{V_{1}}, \hat{G}^{V_{1}} \hat{\eta}_{V_{1}}\right\rangle-2\left\langle\hat{\eta}_{V_{1}}, \theta_{V_{1}}\right\rangle\right)} \frac{\mathbb{1}_{H_{V_{1}, V_{1}}>0}}{\sqrt{\operatorname{det} H_{V_{1}, V_{1}}}} \prod_{i \in V_{1}} \theta_{i} d \beta_{V_{1}}
$$

is the marginal distribution of $\left(\beta_{i}\right)_{i \in V_{1}}$, and the second term,

$$
\left(\frac{2}{\pi}\right)^{\left|V_{2}\right| / 2} e^{-\frac{1}{2}\left(\left\langle\theta_{V_{2}}, \check{H}^{V_{2}} \theta_{V_{2}}\right\rangle+\left\langle\check{\eta}_{V_{2}}, \check{G}^{V_{2}} \check{\eta}_{V_{2}}\right\rangle-2\left\langle\check{\eta}_{V_{2}}, \theta_{V_{2}}\right\rangle\right)} \frac{\mathbb{1}_{\check{H}^{V_{2}}>0}}{\sqrt{\operatorname{det} \check{H}^{V_{2}}}} \prod_{i \in V_{2}} \theta_{i} d \beta_{V_{2}}
$$

is the conditional density of $\left(\beta_{i}\right)_{i \in V_{2}}$ given the values of $\left(\beta_{j}\right)_{j \in V_{1}}$.

\section{Appendix B: Proof of Ward identities}

Let us prove the first Ward identity. Note the $\nu^{W, \theta, \eta}$ is a probability density for any parameters, in particular true for $\nu^{W, \sqrt{k+\theta^{2}}, \eta}$, now (3.9) is equivalent to the fact that $\nu^{W, \sqrt{k+\theta^{2}}, \eta}$ is a probability, since

$$
\sum_{i \in V} k_{i} \beta_{i}+\frac{1}{2}\langle\theta, H \theta\rangle=\frac{1}{2}\left\langle\sqrt{k+\theta^{2}}, H \sqrt{k+\theta^{2}}\right\rangle+\sum_{i, j} W_{i, j}\left(\sqrt{\left(k_{i}+\theta_{i}^{2}\right)\left(k_{j}+\theta_{j}^{2}\right)}-\theta_{i} \theta_{j}\right) .
$$

For the second Ward identity, set $V_{2}=\left\{i_{0}\right\}$ and $V_{1}=V \backslash\left\{i_{0}\right\}$. Note that

$$
\Xi(k, W, \beta)=\frac{G\left(i_{0}, l\right)}{G\left(i_{0}, i_{0}\right)} \exp \left(-\frac{k_{i_{0}}}{2}\left\langle W_{i_{0}, \cdot}, \hat{G}^{V_{1}} W_{i_{0}, \cdot}\right\rangle-\frac{1}{2}\langle\sqrt{k}, 2 \beta \sqrt{k}\rangle_{V_{1}}\right) .
$$


The factorization and the change of coordinates give

$$
\begin{aligned}
& \nu^{W, \theta, 0}(d \beta)=\mathbb{1}_{\gamma>0} e^{-\theta_{i_{0}}^{2} \gamma} \frac{\theta_{i_{0}}}{\sqrt{\pi \gamma}} d \gamma . \\
& \mathbb{1}_{\hat{H}>0} \sqrt{\frac{2}{\pi}} e^{-\frac{1}{2}\langle\theta, 2 \beta \theta\rangle_{V_{1}}+\frac{1}{2}\left\langle\theta, \Delta_{W} \theta\right\rangle_{V_{1}}-\frac{1}{2} \theta_{i_{0}}^{2}\left\langle W_{i_{0}}, \cdot, \hat{G}^{V_{1}} W_{i_{0}}, \cdot\right\rangle+\theta_{i_{0}}\left\langle\theta, W_{i_{0}}, \cdot\right\rangle_{V_{1}}} \frac{1}{\sqrt{\operatorname{det} \hat{H}}} \prod_{i \in V_{1}} \theta_{i} d \beta_{i}
\end{aligned}
$$

Therefore, we see that $\Xi(k, W, \beta)$ plug in well in $\nu^{W, \theta, 0}$. More precisely, we can write (note that $\frac{G\left(i_{0}, l\right)}{G\left(i_{0}, i_{0}\right)}=\sum_{k \sim i_{0}} W_{i_{0}, k} \hat{G}^{V_{1}}(k, l)$ which is independent of $\gamma$ )

$$
\begin{aligned}
& \langle\Xi(k, W, \beta)\rangle_{W, \theta, 0}=\int_{\gamma>0} e^{-\theta_{i_{0}}^{2} \gamma} \frac{\theta_{i_{0}}}{\sqrt{\pi \gamma}} d \gamma \cdot \int_{\hat{H}>0} \frac{G\left(i_{0}, l\right)}{G\left(i_{0}, i_{0}\right)} \sqrt{\frac{2}{\pi}}|V|-1 \\
& e^{-\frac{1}{2}\left\langle\sqrt{\theta^{2}+k}, 2 \beta \sqrt{\theta^{2}+k}\right\rangle_{V_{1}}-\frac{1}{2}\left(\theta_{i_{0}}^{2}+k_{i_{0}}\right)\left\langle W_{i_{0},}, \hat{G}^{V_{1}} W_{i_{0}}, \cdot\right\rangle} \frac{1}{\sqrt{\operatorname{det} \hat{H}}} \prod_{i \neq i_{0}} \theta_{i} d \beta_{i} .
\end{aligned}
$$

As $\gamma$ is independent of the rest in (8.3), using the fact that $\nu^{W, \sqrt{\theta^{2}+k}, 0}$ is a probability measure and

$$
\left\langle\frac{G\left(i_{0}, l\right)}{G\left(i_{0}, i_{0}\right)}\right\rangle_{W, \sqrt{\theta^{2}+k}, 0}=\sqrt{\frac{\theta_{l}^{2}+k_{l}}{\theta_{i_{0}}^{2}+k_{i_{0}}}}
$$

we deduce the second Ward identity.

Equation (8.4) is well known, it first appear in Equation (B.3) of [DSZ10] (where $\theta$ and $k$ are constant), as a consequence of supersymmetric localization. However, to be self-contained, we provide a non supersymmetric proof of it. The idea is to again to use symmetry. It is enough to prove (8.4) for the case $k \equiv 0$. We start by proving the following

$$
\int_{\hat{H}>0} \sqrt{\frac{2}{\pi}}^{|V|-1} e^{-\frac{1}{2} \sum_{i \sim j} W_{i, j}\left(\frac{G\left(i_{0}, j\right)}{G\left(i_{0}, i\right)} \theta_{i}^{2}+\frac{G\left(i_{0}, i\right)}{G\left(i_{0}, j\right)} \theta_{j}^{2}-2 \theta_{i} \theta_{j}\right)} \frac{1}{\sqrt{\operatorname{det} \hat{H}}} \prod_{i \neq i_{0}} \theta_{i} d \beta_{i}=1,
$$

where the sum $\sum_{i \sim j}$ is over all non oriented edges with end points in $V$. We obtain (8.5) from the second line of Equation (8.2) as follows. First integrate out the variable $\gamma$ in (8.2), then use (3.8) and (3.7). We perform the change of variable

$$
\left(\beta_{i}\right)_{i \neq i_{0}} \mapsto\left(u_{i}\right)_{i \neq i_{0}}, \text { where } e^{u_{i}}=\frac{G\left(i_{0}, i\right)}{G\left(i_{0}, i_{0}\right)}\left(\prod_{j \neq i_{0}} \frac{G\left(i_{0}, j\right)}{G\left(i_{0}, i_{0}\right)}\right)^{-1 /|V|}, i \in V,
$$

in particular $\sum_{i \in V} u_{i}=0$. The change of variables leads to

$$
\begin{aligned}
& \int_{\sum_{i \in V} u_{i}=0} \sqrt{\frac{2}{\pi}}^{|V|-1}\left(\prod_{i \neq i_{0}} \theta_{i}\right) e^{u_{i}} e^{-\frac{1}{2} \sum_{\{i, j\} \in E} W_{i, j}\left(e^{u_{i}-u_{j}} \theta_{j}^{2}+e^{u_{j}-u_{i}} \theta_{i}^{2}-2 \theta_{i} \theta_{j}\right)} \\
& \times \sqrt{D(W, u)} \prod_{i \neq i_{0}} d u_{i}=1
\end{aligned}
$$

where $D(W, u)=\sum_{\text {spanning tree } T} \prod_{\{i, j\} \in T} W_{i, j} e^{u_{i}+u_{j}}$. The integral equals 1 for any $W, \theta$, more importantly, for any $i_{0}$ (as in the definition $\gamma=1 /\left(2 G\left(i_{0}, i_{0}\right)\right)$, $i_{0}$ is arbitrary). Notice that $\frac{G\left(i_{0}, l\right)}{G\left(i_{0}, i_{0}\right)}=e^{u_{l}-u_{i_{0}}}$ and

$$
\left(\prod_{i \neq i_{0}} \theta_{i}\right) e^{u_{i_{0}}} \cdot e^{u_{l}-u_{i_{0}}}=\frac{\theta_{l}}{\theta_{i_{0}}} \cdot\left(\prod_{i \neq l} \theta_{i}\right) e^{u_{l}}
$$


VRJP and fractional moment localization

Hence

$$
\begin{aligned}
& \left\langle\frac{G\left(i_{0}, l\right)}{G\left(i_{0}, i_{0}\right)}\right\rangle_{W, \theta, 0} \\
& =\frac{\theta_{l}}{\theta_{i_{0}}} \int_{\sum_{i \in V} u_{i}=0} \sqrt{\frac{2}{\pi}}^{|V|-1}\left(\prod_{i \neq l} \theta_{i}\right) e^{u_{l}} e^{-\frac{1}{2} \sum_{\{i, j\} \in E} W_{i, j}\left(e^{u_{i}-u_{j}} \theta_{j}^{2}+e^{u_{j}-u_{i}} \theta_{i}^{2}-2 \theta_{i} \theta_{j}\right)} \\
& \times \sqrt{D(W, u)} \prod_{i \neq i_{0}} d u_{i}=\frac{\theta_{l}}{\theta_{i_{0}}}
\end{aligned}
$$

where in the last step we used (8.6) with $i_{0}$ replaced with $l$.

\section{References}

[ACK14] Omer Angel, Nicholas Crawford, and Gady Kozma. Localization for linearly edge reinforced random walks. Duke Mathematical Journal, 163(5):889-921, 2014. MR3189433

[AM93] Michael Aizenman and Stanislav Molchanov. Localization at large disorder and at extreme energies: An elementary derivations. Communications in Mathematical Physics, 157(2):245-278, 1993. MR-1244867

[BHS18] Roland Bauerschmidt, Tyler Helmuth, and Andrew Swan. Dynkin isomorphism and mermin-wagner theorems for hyperbolic sigma models and recurrence of the twodimensional vertex-reinforced jump process. arXiv preprint arXiv:1802.02077, 2018. MR-4021254

[BS10] Anne-Laure Basdevant and Arvind Singh. Continuous-time vertex reinforced jump processes on galton-watson trees. Annals of Applied Probability, 22, 05 2010. MR2985176

[CD87] Don Coppersmith and Persi Diaconis. Random walk with reinforcement. Unpublished manuscript, pages 187-220, 1987.

[Col09] Andrea Collevecchio. Limit theorems for vertex-reinforced jump processes on regular trees. Electron. J. Probab., 14:1936-1962, 2009. MR-2540854

[CZ18] Xinxin Chen and Xiaolin Zeng. Speed of vertex-reinforced jump process on galtonwatson trees. Journal of Theoretical Probability, 31(2):1166-1211, 2018. MR-3803928

[DV02] Burgess Davis and Stanislav Volkov. Continuous time vertex-reinforced jump processes. Probability theory and related fields, 123(2):281-300, 2002. MR-1900324

[DV04] Burgess Davis and Stanislav Volkov. Vertex-reinforced jump processes on trees and finite graphs. Probability theory and related fields, 128(1):42-62, 2004. MR-2027294

[DR06] Persi Diaconis and Silke WW Rolles. Bayesian analysis for reversible Markov chains. The Annals of Statistics, pages 1270-1292, 2006. MR-2278358

[DMR17a] Margherita Disertori, Franz Merkl, and Silke W. W. Rolles. A supersymmetric approach to martingales related to the vertex-reinforced jump process. Lat. Am. J. Probab. Math. Stat, 2017. MR-3663098

[DMR17b] Margherita Disertori, Franz Merkl, and Silke WW Rolles. Martingales and some generalizations arising from the supersymmetric hyperbolic sigma model. arXiv preprint arXiv:1710.02308, 2017. MR-3903028

[DS10] Margherita Disertori and Tom Spencer. Anderson localization for a supersymmetric sigma model. Communications in Mathematical Physics, 300(3):659-671, 2010. MR2736958

[DSZ10] Margherita Disertori, Tom Spencer, and Martin R Zirnbauer. Quasi-diffusion in a 3D supersymmetric hyperbolic sigma model. Communications in Mathematical Physics, 300(2):435-486, 2010. MR-2728731

[KR00] Michael S Keane and Silke WW Rolles. Edge-reinforced random walks on finite graphs. Verhandelingen KNAW, 52, 2000. MR-1832379 
VRJP and fractional moment localization

[LW17] Gérard Letac and Jacek Wesołowski. Multivariate reciprocal inverse gaussian distributions from the Sabot-Tarrès-Zeng integral. arXiv preprint arXiv:1709.04843, 2017.

[MR09] Merkl, Franz and Rolles, Silke WW Edge-reinforced random walk on one-dimensional periodic graphs Probability theory and related fields, 145(3-4):323-249, 2009. MR2529432

[MÖR08] Franz Merkl, Aniko Öry, and Silke WW Rolles. The 'magic formula'for linearly edgereinforced random walks. Statistica Neerlandica, 62(3):345-363, 2008. MR-2441859

[MRT16] Franz Merkl, Silke WW Rolles, and Pierre Tarrès. Convergence of vertex-reinforced jump processes to an extension of the supersymmetric hyperbolic nonlinear sigma model. arXiv preprint arXiv:1612.05409, 2016. MR-4087496

[PB74] Poole, George, and Thomas Boullion. A Survey on M-Matrices. SIAM Review, vol. 16, no. 4, 1974, pp. 419-427. MR-0352146

[RN18] Olivier Raimond and Tuan-Minh Nguyen. Strongly vertex-reinforced jump process on a complete graph. arXiv e-prints, page arXiv:1810.06905, Oct 2018.

[ST15a] Christophe Sabot and Pierre Tarrès. Edge-reinforced random walk, vertex-reinforced jump process and the supersymmetric hyperbolic sigma model. J. Eur. Math. Soc., 17(9):2353-2378, 2015. MR-3420510

[ST15b] Christophe Sabot and Pierre Tarres. Inverting Ray-Knight identity. Prob. Th. Rel. Fields, online first, 2015. MR-3520013

[STZ17] Christophe Sabot, Pierre Tarrès, and Xiaolin Zeng. The vertex reinforced jump process and a random schrödinger operator on finite graphs. The Annals of Probability, 45(6A):3967-3986, 2017. MR-3729620

[SZ19] Christophe Sabot and Xiaolin Zeng. A random schrödinger operator associated with the vertex reinforced jump process on infinite graphs. Journal of the American Mathematical Society, 32(2):311-349, 2019. MR-3904155

[Zen16] Xiaolin Zeng. How vertex reinforced jump process arises naturally. In Annales de l'Institut Henri Poincaré, Probabilités et Statistiques, volume 52, pages 1061-1075. Institut Henri Poincaré, 2016. MR-3531700

[Zir91] Martin R Zirnbauer. Fourier analysis on a hyperbolic supermanifold with constant curvature. Communications in mathematical physics, 141(3):503-522, 1991. MR1134935

Acknowledgments. X. Z. want to thank R. Peled for his encouragement of writing this paper. We thank an anonymous referee for very helpful comments. 